\title{
Signatures of an Encounter Between the G2 Cloud and a Jet from Sgr A*
}

\author{
Farhad Yusef-Zadeh ${ }^{1}$ and Mark Wardle ${ }^{2}$ \\ ${ }^{1}$ Department of Physics and Astronomy and Center for Interdisciplinary Research in \\ Astronomy, Northwestern University, Evanston, IL 60208 \\ ${ }^{2}$ Department of Physics and Astronomy and Research Center for Astronomy, Astrophysics \\ E Astrophotonics, Macquarie University, Sydney NSW 2109, Australia
}

\begin{abstract}
The recent discovery of the G2 cloud of dense, ionized gas on a trajectory toward Sgr A*, the black hole at the dynamical center of the Galaxy, offers a unique opportunity to observe an accretion event onto a massive black hole as well as to probe its immediate environment. Simulations and models predict increased X-ray and radio variability resulting from increased accretion driven by drag on an atmosphere of hot, X-ray emitting gas surrounding Sgr A*. Here, we present $\mathrm{X}$-ray and radio light curves of the emission resulting from the potential encounter of the G2 cloud with a relativistic jet from Sgr A*. This interaction would violently shock a portion of the G2 cloud to temperatures $\sim 10^{8} \mathrm{~K}$ resulting in bright X-ray emission from the dense, shocked gas as it adiabatically expands. The 2-10 keV luminosity may reach $\sim 10$ times the quiescent $\mathrm{X}$-ray flux of Sgr $\mathrm{A}^{*}$. approximately $3 \mathrm{~L}_{\odot}$ is emitted above $10 \mathrm{keV}$ at the peak of the light curve, with significant softening of the spectrum occurring as the gas subsequently cools. Observations with NuSTAR would therefore be able to confirm such an event as well as determine the cloud speed. At radio wavelengths, the associated synchrotron radio emission may reach levels of a few Jy.
\end{abstract}

Subject headings: Galaxy: center - galaxies: active - ISM: jets and outflows black hole physics

\section{Introduction}

Recent observations of the Galactic center at near-IR wavelengths have identified a dense, dusty gas cloud (dubbed "G2" by Gillessen et al 2012, 2013; see also Phifer et al. 2013 ) on a collision course with the compact radio source Sgr $A^{*}$, the $4.3 \times 10^{6} \mathrm{M}_{\odot}$ black 
hole located at the dynamical center of our Galaxy (e.g. Ghez et al. 2008; Gillessen et al. 2009). The cloud's mass is estimated to be $\sim 3$ Earth masses with electron density $\sim 2.6 \times 10^{5}$ $\mathrm{cm}^{-3}$, and dust and gas temperatures of $\sim 600 \mathrm{~K}$, and $\sim 10^{4} \mathrm{~K}$, respectively. $\mathrm{G} 2$ is on a highly eccentric orbit (e 0.97) that is coplanar with the clockwise stellar disk (Paumard et al. 2006; Lu et al. 2009; Bartko et al. 2009) and is expected to reach its pericenter distance of $\sim 2000$ Schwarzschild radii $\left(\mathrm{R}_{S}\right)$ from Sgr $A^{*}$ in mid September, 2013 (Gillessen et al. 2013) or March 2014 (Phifer et al. 2013).

The origin and nature of the G2 cloud is unclear - models include a pressure-confined compact gas cloud (Gillessen et al. 2012), an extended spherical shell created by colliding stellar winds (Burkert et al. 2012; Schartmann et al. 2012), a photo-evaporative wind from a proto-planetary disk surrounding a low-mass star (Murray-Clay \& Loeb 2012), a shell from a nova outburst (Meyer \& Meyer-Hofmeister 2012), or an outflow from a T Tauri star (Scoville \& Burkert 2013), in which case the observed emission arises from the ionized surface of a more extensive cloud. The change in the kinematic structure of the emission, however, is consistent with simple tidal stretching, and the mass of ionized gas inferred from the $\operatorname{Br} \gamma$ emission has not changed significantly over 4 years of monitoring, casting doubt on some models.

G2 is being tidally sheared (Gillesen et al. 2013) as it approaches pericenter, and will eventually be disrupted. Shredding of the cloud due to drag on the hot, tenuous medium thought to be responsible for the X-ray emission within $0.5^{\prime \prime}$ of $\operatorname{Sgr} \mathrm{A}^{*}$ may hasten this and lead to the accretion of a substantial fraction of G2 on to the black hole (e.g. Gillesen et al 2012; Burkert et al. 2012; Schartman et al 2012; but see also Anninis et al 2012 and Saitoh et al 2012). This interaction is also expected to produce a weak bow shock in the pre-existing hot quasi-vitalized gas settling in Sgr $\mathrm{A}^{*}$. Electrons accelerated in the shock may increase the radio flux associated with Sgr $\mathrm{A}^{*}$ by an order of magnitude (Narayan et al. 2012; Sadowski et al. 2013). In addition, Bartos et al. (2013) have discussed the accretion of some of G2 by a population of stellar black holes and neutron stars that may be residing close to the dynamical center of the Galaxy because of mass segregation in the inner nuclear cluster centered on Sgr A*.

Here, we point out that G2 may also interact with a jet emerging from Sgr A* as has been tentatively detected by Yusef-Zadeh at al 2012, and previously proposed to explain the SED and the variability in the emission from Sgr A* (e.g. Markoff et al. 2007, Falcke et al. 2009).

The broad-band spectrum of the quiescent emission from Sgr A* has been modeled in terms of emission from the base of a relativistic jet, and Very Long Baseline Array measurements suggest that the position angle $(\mathrm{PA})$ of the outflowing material is $\sim 90^{0}$ (Markoff et 
al. 2007). Short time scale variability of Sgr $\mathrm{A}^{*}$ and the time delay between the peaks of radio emission at different frequencies has also been interpreted in the the context of a jet or outflow from Sgr A* (Yusef-Zadeh et al. 2006; Maitra et al. 2009). These measurements suggest a mass outflow rate $\lesssim 2 \times 10^{-8} \mathrm{M}_{\odot} \mathrm{yr}^{-1}$.

On larger, but sub-parsec scales, a number of observations indicate a collimated outflow or a jet from Sgr A*. A chain of radio blobs links Sgr A* to the "minicavity" at $8.4 \mathrm{GHz}$ (Yusef-Zadeh, Morris \& Ekers 1990; Wardle \& Yusef-Zadeh 1992). The minicavity, roughly at $\mathrm{PA} \sim 60^{0}$ from Sgr $\mathrm{A}^{*}$, shows enhanced FeIII line emission, a high electron temperature and is kinematically disturbed (Eckart et al. 1990; Lutz et al. 1993). Infrared observations have also discovered two dust features with cometary morphology along the line between Sgr $A^{*}$ and the mini-cavity (Muzic et al. 2010), their orientation is suggestive of an outflow from young massive stars or from Sgr A* (Lutz et al. 1993; Muzic et al. 2009).

Radio observations of Sgr A* have provided a tantalizing detection of a jet-like linear feature emanating symmetrically from Sgr A* on a scale of $\sim 3 \mathrm{pc}$ (Yusef-Zadeh et al. 2012). A number of radio continuum features with X-ray and FeII line counterparts, and linearly polarized features are also detected along the axis of this $3 \mathrm{pc}$ jet feature. The feature is projected at an angle that appears to be parallel to the angular momentum axis of the clockwise stellar disk and the orbital plane of G2. The axis of the jet from Sgr A* is estimated to be at $\mathrm{PA} \sim 60^{\circ}$. Model fitting of the polarization of the near-IR emission from Sgr $\mathrm{A}^{*}$ infers the spin axis of Sgr A* to be similar to the PA of the jet (Zamaninasab et al. 2011). Based on the interaction of the jet and the minicavity, the mass outflow rate in the jet is estimated to be $10^{-6} \mathrm{M}_{\odot} \mathrm{yr}^{-1}$, with a Lorentz factor $\gamma \sim 3$.

This motivates us to investigate potential signatures of the interaction of a jet emanating from Sgr $A^{*}$ and the G2 cloud. The collimated appearance of the jet on parsec scales suggest that the jet close to Sgr $\mathrm{A}^{*}$ must be narrow with a diameter of $d_{j} \sim 3000 R_{S}$. Figure 1 shows a schematic diagram of the geometry of the jet and G2 as they interact with each other. In this scenario, the G2 cloud is assumed to be tidally stretched as it sweeps across the barrel of the jet. Assuming that the ram pressure in the jet greatly exceeds that associated with the orbital motion of G2 (i.e. the outflow rate $\geq 10^{-9} \mathrm{M}_{\odot} \mathrm{yr}^{-1}$ ), the jet is not significantly deflected by the interaction, but instead acts as a solid obstacle. As the orbital speed of G2 is hundreds of times its internal sound speed, a bow shock standing off from the jet forms within G2 as it streams past, as sketched in Figure 1. The hot $\left(T \sim 10^{8} \mathrm{~K}\right)$ and dense $\left(n_{\mathrm{H}} \sim 10^{6} \mathrm{~cm}^{-3}\right)$ shocked gas emits X-rays, but cools primarily adiabatic expansion parallel to the jet axis into the under pressured external medium. Relativistic electrons are accelerated in the bow shock, producing synchrotron emission. 


\section{Estimates and light curves}

Before computing model light curves for this interaction, we first adopt nominal values of the critical physical parameters and estimate the strength of the emission expected in X-rays and radio. Before being tidally sheared G2 had a diameter $\sim 300 R_{S}$ and number density of hydrogen nuclei $3 \times 10^{5} \mathrm{~cm}^{-3}$, corresponding to a mass $10^{-5} \mathrm{M}_{\odot}$ (Gillesen et al 2011, 2012). G2 will be tidally sheared along its orbit and compressed perpendicular to it (e.g. Saitoh et al. 2012). Here we assume that at the point of interaction with the jet, the cloud is stretched to a length $L_{g}=10^{4} R_{S}$, while its transverse dimension is $L_{j}=10^{3} R_{S}$. This combination of dimensions implies that the cloud density is very close to its initial density, so we simply adopt $n_{g}=3 \times 10^{5} \mathrm{~cm}^{-3}$ as the hydrogen density. The temperature in the cloud is maintained close to $10^{4} \mathrm{~K}$ by efficient cooling (Saitoh et al. 2012). The sound speed is therefore $\approx 10 \mathrm{~km} \mathrm{~s}^{-1}$. At pericenter the orbital velocity is very close to the escape velocity, ie $6400 \mathrm{~km} \mathrm{~s}^{-1}$. We choose a lower nominal shock speed, $v_{g}=3000 \mathrm{~km} \mathrm{~s}^{-1}$, partly because interaction may occur prior to or subsequent to periastron, and because the cloud velocity may not be perpendicular to the jet. The final quantity that we require is the diameter of the jet, which we set at $d_{j}=300 R_{S}$. This implies an opening angle of about $8^{\circ}$, consistent with estimates based on tentative detection at radio wavelengths (Yusef-Zadeh et al 2012).

Now we consider the emission from the immediate post shock gas, which has a temperature $T=3 \mu v_{g}^{2} / 16 k=1.25 \times 10^{8} \mathrm{~K}$, where $\mathrm{k}$ is Boltzmann's constant and $\mu$ is the mean particle mass, number density $n_{H}=4 n_{g}=1.2 \times 10^{6} \mathrm{~cm}^{-3}$, and emits X-rays with a radiative power per unit volume $\Lambda=n_{\mathrm{H}}^{2} f(T)$ where we approximate the results of Böhringer \& Hensler (1989) for gas of twice solar metallicity by

$f(T) \approx 1.5 \times 10^{-23}\left(\left(T / 4 \times 10^{6}\right)^{-2.75}+\left(T / 4 \times 10^{6}\right)^{0.8}\right) \mathrm{erg} \mathrm{s}^{-1} \mathrm{~cm}^{3}$ when $2 \times 10^{6}<T<10^{7} \mathrm{~K}$ and $f(T) \approx 1.2 \times 10^{-23}\left(\left(T / 1.5 \times 10^{7}\right)^{-1.4}+\left(T / 4 \times 10^{7}\right)^{0.5}\right) \mathrm{erg} \mathrm{s}^{-1} \mathrm{~cm}^{3}$ when $\mathrm{T}>10^{7} \mathrm{~K}$. The shock interaction creates thermal energy at a rate

$$
L_{\text {shock }}=\frac{1}{2} \rho_{g} v_{g}^{3} L_{j} d_{j} \approx 1200 \mathrm{~L}_{\odot} .
$$

over a period

$$
t_{\mathrm{int}}=\frac{L_{g}}{v_{g}}=1.3 \mathrm{yr} .
$$

The radiative timescale for the shocked gas,

$$
t_{\mathrm{rad}}=\frac{5}{2} \frac{k T}{\rho f(T)} \approx 72 \mathrm{yr}
$$

is long compared to its timescale for expansion,

$$
t_{\text {exp }}=\frac{L_{j}}{c_{s}}=0.24 \mathrm{yr} .
$$


so the gas cools by adiabatic expansion. Thus the peak luminosity is approximately the power radiated by the hot gas generated by the shock over an expansion time scale. The thermal energy in this gas is approximately $L_{\text {shock }} t_{\text {exp }}$, yielding an estimate

$$
L_{X} \approx \frac{L_{\text {shock }} t_{\text {exp }}}{t_{\text {rad }}} \approx 4.7 \mathrm{~L} \odot_{\odot}
$$

This luminosity exceeds the $\sim 2.4 \times 10^{33} \mathrm{erg} \mathrm{s}^{-1}$ quiescent X-ray luminosity of Sgr A* based on Chandra observations (Baganoff et al. 2003), so should be detectable with the Chandra, XMM, and Swift observatories.

We adopt an analogous approach to estimating the radio flux arising from synchrotron emission from relativistic electrons accelerated by the shock. We assume that the power that the shock wave deposits into relativistic electrons is $\epsilon L_{\text {shock}}$, with a nominal efficiency $\epsilon=0.011$, and that the electrons are produced with an $E^{-2}$ spectrum. Here the relevant radiative cooling time is the synchrotron loss time scale for the electrons that dominate the emission at, say, $\nu=1.4 \mathrm{GHz}$, so we also need estimate the post shock field strength, which we parametrize as $B^{2}=8 \pi \epsilon_{B} \rho_{g} v_{g}^{2}$. In supernova remnant (SNR) shocks the magnetic field is amplified by cosmic ray acceleration to levels $\epsilon_{B} \sim 10^{-2}-10^{-3}$ (Vink 2012; Helder et al 2012; Ellisen et al 2012), so we adopt $\epsilon_{B}=0.01$, yielding $B \approx 0.12 \mathrm{G}$. These electrons have energy $E_{\nu} \approx 26 \mathrm{MeV}$, with loss time

$$
t_{\mathrm{synch}} \approx 20 \mathrm{yr} \text {. }
$$

Again, the radiative loss time is long, so that we can use a similar expression as for the X-rays to estimate the synchrotron luminosity, and then use $L_{\text {synch }} \approx 4 \pi d^{2} \nu S_{\nu}$ to find the flux, yielding

$$
S_{\nu} \approx \frac{1}{4 \pi d^{2} \nu} \frac{\varepsilon L_{\text {shock }} t_{\text {exp }}}{t_{\text {synch }}} \approx 5.1 \mathrm{Jy} .
$$

which significantly exceeds the $\sim 1$ Jy quiescent flux from Sgr A* at $1.4 \mathrm{GHz}$.

To compute approximate X-ray light curves, we characterize the shocked gas by its crosssectional area $A$, density, temperature, and velocity $v$ perpendicular to $A$. These quantities all evolve as the gas moves downstream. The cross-section expands at a rate $d A / d t=L_{j} c_{s}$ where $c_{s}$ is the adiabatic sound speed, and the associated drop in density and increase in velocity are found by conservation of mass and momentum and noting that the expansion is adiabatic, so that $\rho A v,\left(\rho v^{2}+P\right) A$, and $P / \rho^{5 / 3}$ are constant. The resulting profile allows us to compute the X-ray emissivity by integrating the volume emissivity of the $2-10 \mathrm{keV}$

\footnotetext{
${ }^{1}$ This is distinct from the fraction $\eta=0.05$ of shocked electrons that are assumed to be accelerated by Narayan et al (2012) and Sadowski et al (2013), equivalent to $\epsilon \approx 2$ and 0.4 , respectively.
} 
X-ray emission, ie $\Lambda[\exp (-2 \mathrm{keV} / k T)-\exp (-10 \mathrm{keV} / k T)]$, as the gas moves downstream. We have not accounted for extinction but this is not expected to be severe as the spectrum is hard $(k T \sim 10 \mathrm{keV})$.

The synchrotron light curves are computed based on the assumption that the field and electrons are advected along with the postshock gas. The magnetic field must be tangled if it is significantly larger than the preshock field, so it's strength scales as $\rho^{2 / 3}$. Meanwhile, the electrons' individual energies scale as $\rho^{1 / 3}$ while preserving their spectral index: as a result the electron and magnetic energy densities both scale as $\rho^{4 / 3}$ and the synchrotron emissivity scales as $\rho^{7 / 3}$.

The resulting X-ray and $1.4 \mathrm{GHz}$ light curves for our fiducial parameters are shown in Figure 2, respectively For our nominal parameters (solid curves), the X-ray light curve (upper panel) begins sharply once shocked gas starts to be created by the interaction of G2 with the jet. The luminosity rises rapidly as the mass of shocked gas increases, but the rate of increase in $L_{X}$ declines once adiabatic expansion starts to cool the oldest gas. During this phase, the net change in the shocked gas is the addition of successively older and cooler layers of gas at the extreme downstream end of the shocked gas, and the change in luminosity corresponds to the emission from this oldest layer. The rise in luminosity terminates once the interaction ends and the cooling gas is no longer being supplemented by newly shocked material. As the luminosity is dominated by this material, the initial decline is sharp, but then tails off as the shocked gas slowly cools off. The peak luminosity is consistent with the rough estimate given by eq (5); the extreme sharpness of the initial decline from the peak reflects the artificially sharp truncation of shock heating in our treatment. The $1.4 \mathrm{GHz}$ light curve behave similarly, as shown in the lower panel of Figure 2. Note that the peak flux density at $1.4 \mathrm{GHz}$ is few times lower than the estimate in eq (7) because significant emission occurs at higher frequencies. Also plotted are the light curves for different choices of shock speed. At lower speeds the X-ray luminosity and synchrotron emission drop sharply, because the shock luminosity depends quadratically on the cloud speed $v_{g}$. At higher speeds, the radio flux increases similarly, but the X-ray flux does not because the gas temperature is so high that much of the X-ray emission emerges at energies exceeding $10 \mathrm{keV}$.

We illustrate this in the top panel of Figure 3 by comparing the luminosities in different $\mathrm{X}$-ray bands between 5 and $80 \mathrm{keV}$ for our nominal shock speed of $3000 \mathrm{~km} / \mathrm{s}$. The peak luminosities between $5-10$ and $20-40 \mathrm{keV}$ are similar because the shocked gas initially has $k T \approx 10 \mathrm{keV}$. Once the interaction between $\mathrm{G} 2$ and the jet ceases, the spectrum becomes softer as the shocked gas cools by adiabatic expansion. The lower panel shows the effect of increasing $v_{g}$ to $5000 \mathrm{~km} \mathrm{~s}^{-1}$. Now the gas is shocked to $k T \approx 25 \mathrm{keV}$, with a corresponding dramatic increase in the power radiated between $20-40$ and $40-80 \mathrm{keV}$ relative to the lower 
energy bands. We conclude that observations with NuSTAR would be ideally suited to determine the gas temperature and determine the shock speed.

\section{Discussion and Conclusion}

We have shown that if the G2 cloud encounters a jet from Sgr A* during its passage around pericenter then it is likely to produce detectable $\mathrm{X}$-ray emission. The X-ray luminosity is far larger than expected from the drag interaction of G2 with a hot medium centered on Sgr $A^{*}$ because of the violence with which the cloud encounters the jet, which shocks a fraction of the cloud to high temperatures and high density. The greatest uncertainties in estimating the luminosity are the density and geometry of the incoming cloud, which may be significantly compressed by the tidal effects of Sgr A* (Saitoh et al 2012). Close to pericenter G2 will be strongly compressed in the direction perpendicular to the orbital plane (Saitoh et al 2012), and the density will increase by orders of magnitude. This increases the emission from the shocked gas, which is proportional to $n_{g}^{2} \mathrm{~L}_{\mathrm{j}}$, but decreases the probability of interaction with the jet. The decays in the curve due to adiabatic expansion is also unique to this model, reflecting the fact that the shocked cloud material is over pressured. Observations using NuSTAR would provide a strong test of this scenario, as the high temperature and subsequent expansion of the shocked gas produces detectable spectral evolution in the X-ray light curves between 5 and $80 \mathrm{keV}$. We also predict a detectable radio flux, based on the assumption that $1 \%$ of the kinetic luminosity is deposited into relativistic electrons and that the magnetic field is amplified to a level similar to that inferred for supernova remnants.

The greatest uncertainty is whether a jet from Sgr A* is sufficiently aligned with the orbital plane of G2 for an interaction to even occur. Figure 4 shows the range of jet inclinations that lie within $5^{\circ}$ of the orbital plane as a function of jet PA. We have plotted the inclination's cosine so that solid angle is directly proportional to area, allowing assessment of probabilities. A randomly-oriented jet has probability $\approx 0.087$ of lying within $5^{\circ}$ of the plane, precisely the fraction of the plot area lying within the target shaded region. The blue rectangles indicate the ranges of jet orientation previously inferred either from Sgr A* emission models or from parsec-scale jet-like features identified in radio and X-rays: (i) combined disk/jet models of the emission at $7 \mathrm{~mm}$ detected using VLBA (MO7 - Markoff et al. 2007), (ii) the normal to the accretion disk orientation inferred from modeling the sub-mm VLBI observations of Sgr A* (B11 - Broderick et al. 2011), (iii) polarization of NIR flaring (Z11Zamaninasab et al. 2011), (iv) a 0.5 pc jet-like feature identified in X-rays (M08 - Muno et al. 2008), and (v) a parsec-scale radio jet (YZ12 - Yusef-Zadeh et al 2012). In the latter two cases, the inclination ranges are very uncertain. We adopt $\pm 45^{\circ}$ from the plane of the sky 
for the X-ray jet. For the radio jet, the $\mathrm{PA} \approx 238^{\circ}$ side of the jet is directed between $30-60^{\circ}$ in front of the plane of the sky so that it can collide with material to form the minicavity (Yusef-Zadeh et al. 2012). The uncertainty in both jet orientation and the orbital plane make a reliable estimate impossible, but given the tendency of the inferred jets to follow the orbit of G2, odds of 1 in 3 or 4 are not implausible.

The center and lower panels of Figure 4 show the shock speed and epoch of a potential interaction as a function of jet PA. The shock speed is equated to the azimuthal component of G2's orbital velocity as this will be normal to the jet. As we expect significant emission only for shock speeds exceeding $2000 \mathrm{~km} / \mathrm{s}$ (unless the density of G2 is significantly enhanced by tidal effects), the relevant range of PA and epoch is +100 to $-100^{\circ}$ and early 2013 - mid2014 for the Gillesen et al (2013) orbit, and $-140^{\circ}$ to $+70^{\circ}$ and mid-2013 - end 2014 for the Phifer et al. (2013) orbit. In either case we expect appreciable emission if the cloud collides with $\mathrm{B} 11 / \mathrm{Z} 11 / \mathrm{Y} 12$ jets at $\mathrm{PA} \sim 60^{\circ}$ and the $\mathrm{M} 07, \mathrm{M} 08$ jets at $\mathrm{PA} \sim 85^{\circ}$.

In summary, we have described a model in which the G2 cloud runs into a relativistic jet from $\mathrm{Sgr} \mathrm{A}^{*}$ and have predicted X-ray, and radio light curves that are distinct from those produced from the collision of G2 with the hot atmosphere of Sgr A*. Monitoring the emission from Sgr A* at hard X-rays using NuStar may be particularly useful in testing the proposed model.

This work is partially supported by grants AST-0807400 from the NSF and DP0986386 from the Australian Research Council.

\section{REFERENCES}

Anninos, P., Fragile, P. C., Wilson, J., \& Murray, S. D. 2012, ApJ, 759, 132

Baganoff, F. K., Maeda, Y., Morris, M. et al. 2003, ApJ, 591, 891

Bartko, H., Martins, F., Trippe, S. et al. 2009, ApJ, 697, 1741

Bartos, I., Haiman, Z., Kocsis, B., \& Marka, S. 2013, ArXiv e-prints, 2013arXiv1301.5235

Böhringer, H. \& Hensler, G. 1989, A\&A, 215, 147

Broderick, A. E., Fish, V. L., Doeleman, S. S., \& Loeb, A. 2011, ApJ, 735, 110

Burkert, A., Schartmann, M., Alig, C., Gillessen, S., Genzel, R., Fritz, T. K., et al. 2012, ApJ, 750, 58 
Eckart, A., Genzel, R., Krabbe, A. et al. 1990, Nature, 355, 526

Ellison, D. C., Patnaude, D. J., Slane, P., \& Raymond, J. 2010, ApJ, 712, 287

Falcke, H., Markoff, S., \& Bower, G. C. 2009, A\&A, 496, 77

Ghez, A. M., Salim, S., Weinberg, N. N. et al. 2008, ApJ, 689, 1044

Gillessen, S., Eisenhauer, F., Trippe, S. et al. 2009, ApJ, 692, 1075

Gillessen, S., Genzel, R., Fritz, T. K., Quataert, E., Alig, C., Burkert, A., et al. 2012, Nature, 481,51

Gillessen, S., Genzel, R., Fritz, T. K., Eisenhauer, F., Pfuhl, O., Ott, T., et al. 2013, ApJ, 763,78

Helder, E. A., Vink, J., Bykov, A. M., Ohira, Y., Raymond, J. C., \& Terrier, R. 2012, Space Sci. Rev., 173, 369

Lu, J. R., Ghez, A. M., Hornstein, S. D. et al. 2009, ApJ, 690, 1463

Lutz, D., Krabbe, A. \& Genzel, R. 1993, ApJ 418, 244

Maitra, D., Markoff, S., \& Falcke, H. 2009, A\&A, 508, L13

Markoff, S., Bower, G. C. \& Falcke, H. 2007, MNRAS, 379, 1519

Meyer, F. \& Meyer-Hofmeister, E. 2012, A\&A, 546, L2

Muno, M. P., Baganoff, F. K., Brandt, W. N., Morris, M. R. \& Starck, J.-L. 2008, ApJ, 673, 251

Murray-Clay, R. A. \& Loeb, A. 2012, Nature Communications, 3, 1049

Muzić, K., Eckart, A., Schödel, R., Buchholz, R., Zamaninasab, M., \& Witzel, G. 2010, A\&A, 521, A13

Narayan, R., Özel, F., \& Sironi, L. 2012, ApJ, 757, L20

Paumard, T., Genzel, R., Martins, F. et al. 2006, ApJ, 643, 1011

Phifer, K., Do, T., Meyer, L., Ghez, A. M., Witzel, G., Yelda, S., Boehle, A., Lu, J. R., Morris, M. R., Becklin, E. E. et al. 2013, (arXiv1304.5280)

Sadowski, A., Sironi, L., Abarca, D., Guo, X., Ozel, F., \& Narayan, R. 2013, ArXiv e-prints, 2013arXiv1301.3906 
Saitoh, T. R., Makino, J., Asaki, Y., Baba, J., Komugi, S., Miyoshi, M., et al. 2012, ArXiv e-prints, 2012arXiv1212.0349

Schartmann, M., Burkert, A., Alig, C., Gillessen, S., Genzel, R., Eisenhauer, F., et al. 2012, ApJ, 755, 155

Scoville, N. \& Burkert, A. 2013, ArXiv e-prints, 2013arXiv1302.6591

Vink, J. 2012, A\&A Rev., 20, 49

Wardle, M., \& Yusef-Zadeh, F. 308, 1992, Nature, 357, 308

Yusef-Zadeh, F., Arendt, R., Bushouse, H., Cotton, W., Haggard, D., Pound, M. W., et al. 2012, ApJ, 758, L11

Yusef-Zadeh, F., Morris, M. \& Eckers, R. D. 1990, Nature, 348, 45

Yusef-Zadeh, F., Roberts, D., Wardle, M., Heinke, C. O., \& Bower, G. C. 2006, ApJ, 650, 189

Zamaninasab, M., Eckart, A., Dovciak, M., Karas, V., Schödel, R., Witzel, G., et al. 2011, MNRAS, 413, 322

Zhao, J.-H., Bower, G. C., \& Goss, W. M. 2001, ApJ, 547, L29 


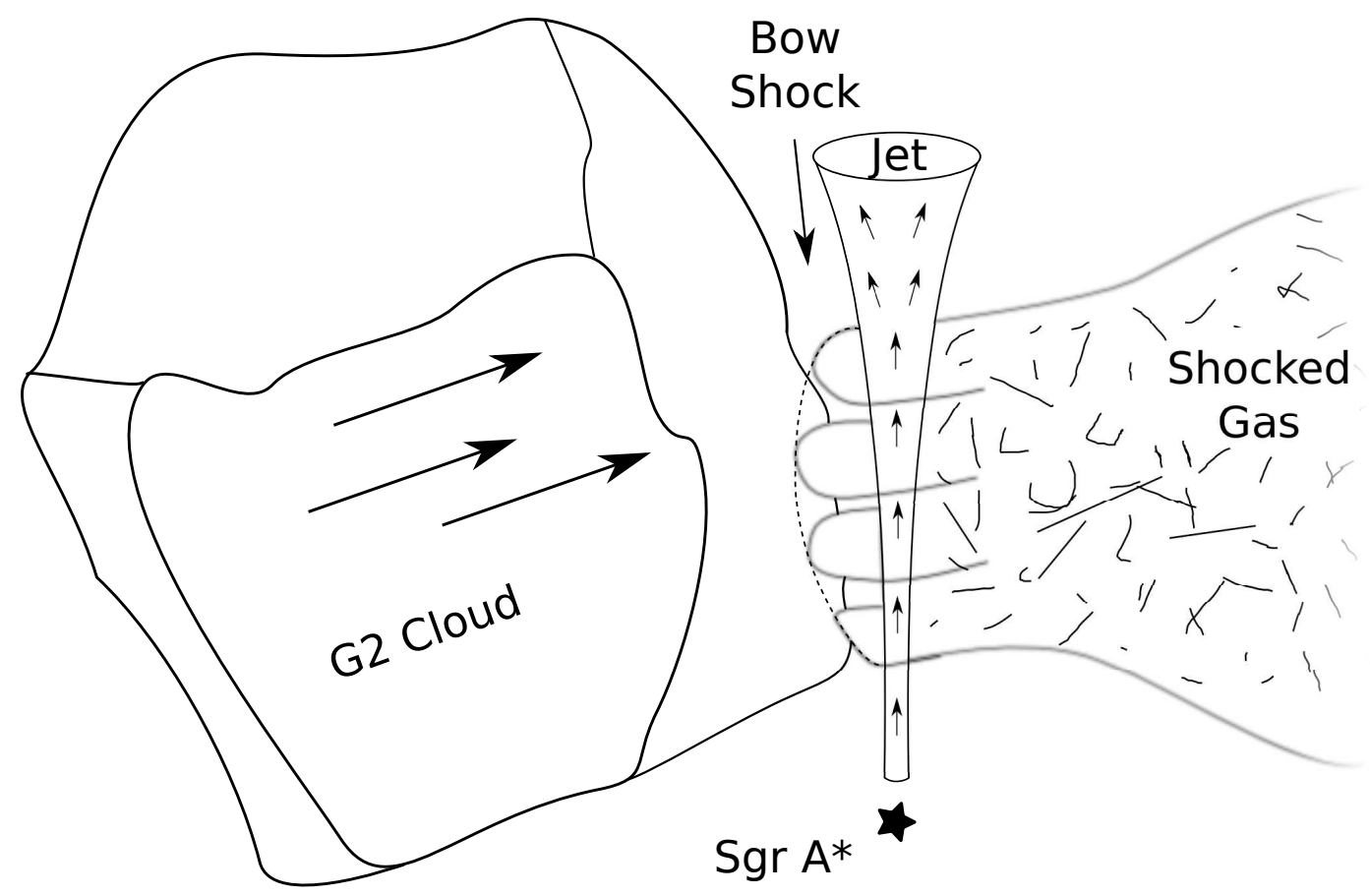

Fig. 1. - A Schematic diagram of a model in which the G2 cloud runs into a relativistic jet emanating from Sgr A*. 

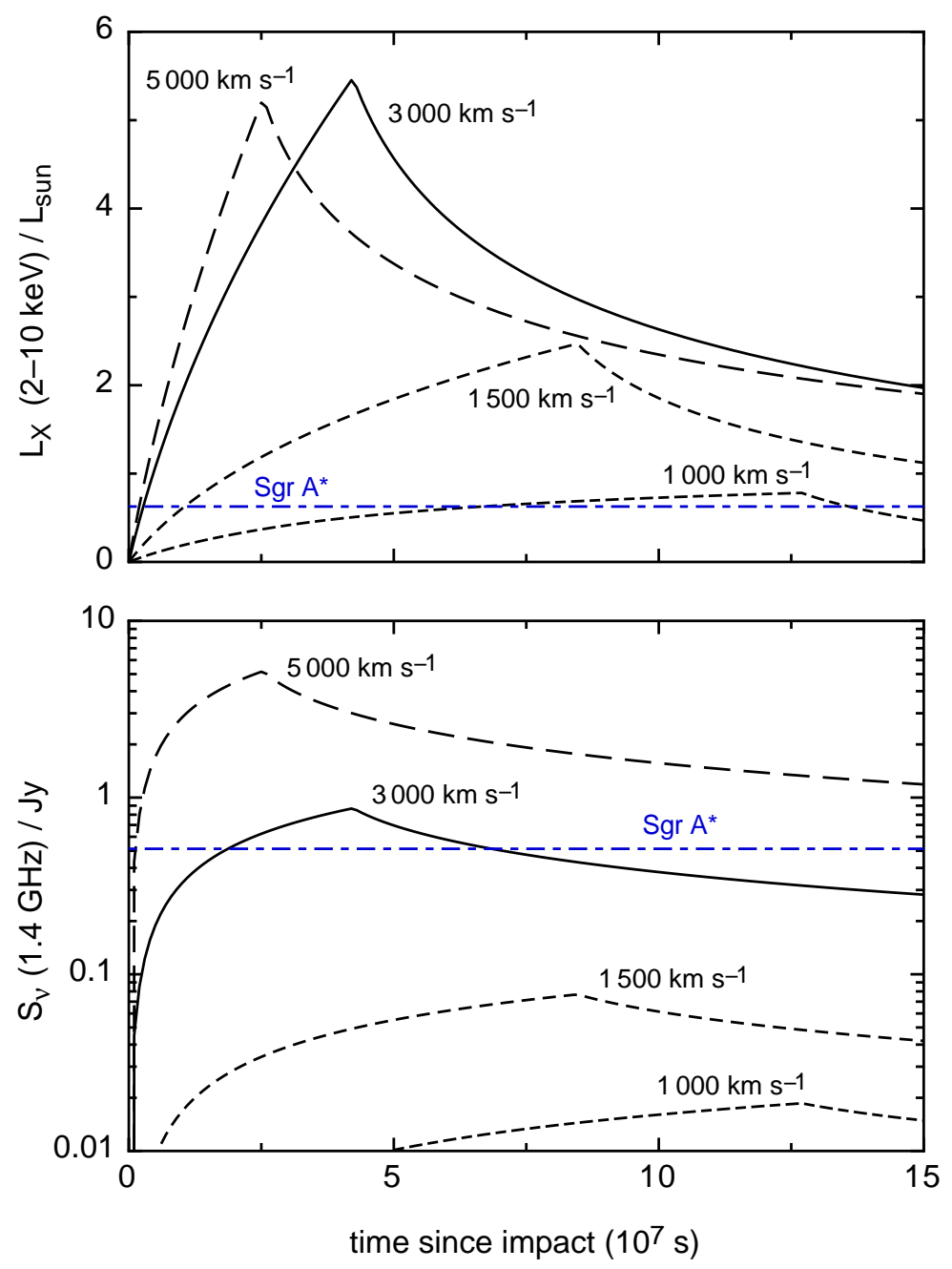

Fig. 2.- X-ray (upper panel) and radio light curves lower panel) arising from the interaction of the cloud with a jet from Sgr A*, for different values of the G2 cloud velocity normal to the jet axis. The blue dot-dashed line in each panel indicates the level of the quasi-steady emission from Sgr A* (Zhao et al 2001; Baganoff et al. 2003). 

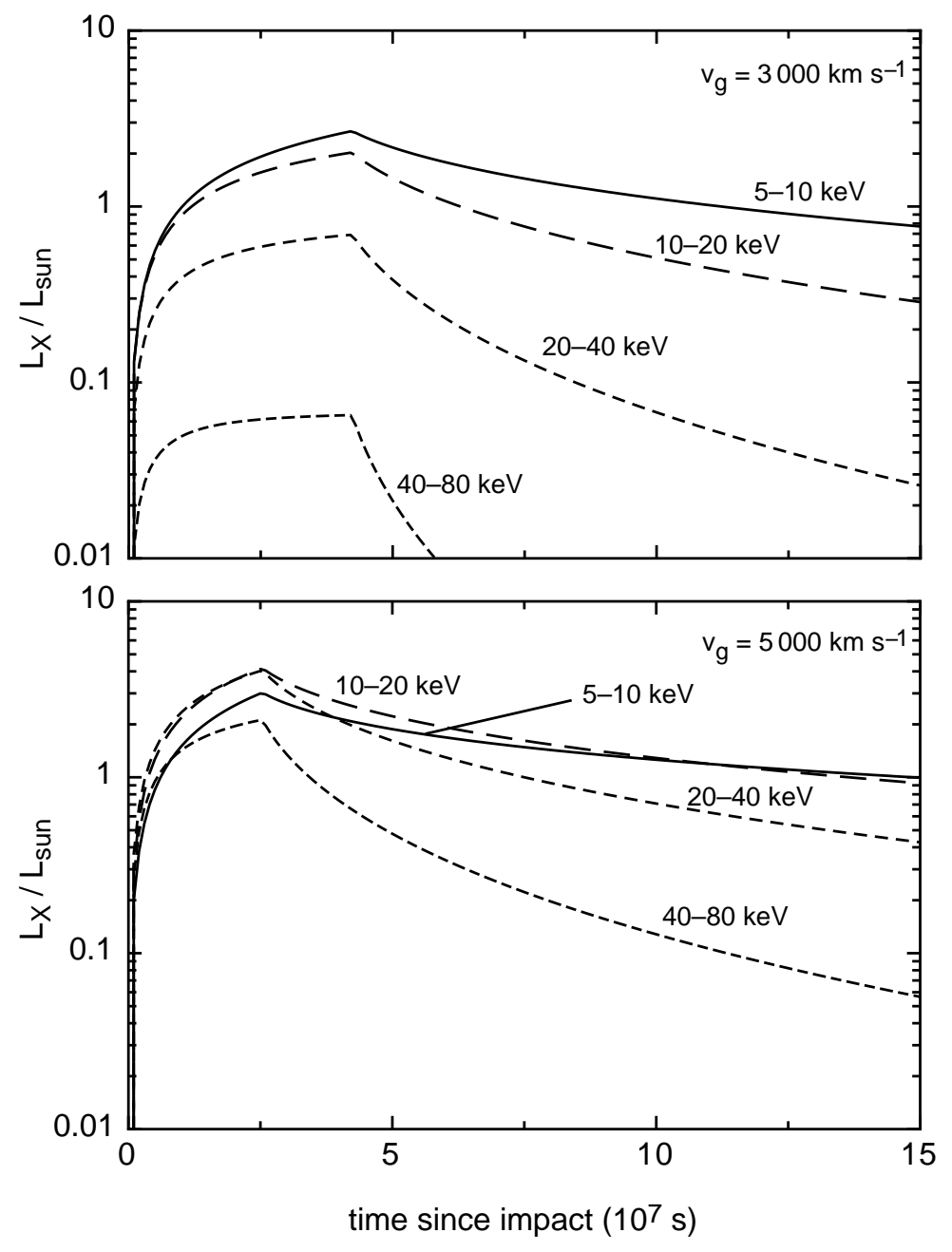

Fig. 3. - Light curves in different X-ray bands arising from the collision of the G2 cloud with a jet from Sgr A* for cloud velocities of $3000 \mathrm{~km} \mathrm{~s}^{-1}$ (top panel) and $5000 \mathrm{~km} \mathrm{~s}^{-1}$ (lower panel). 

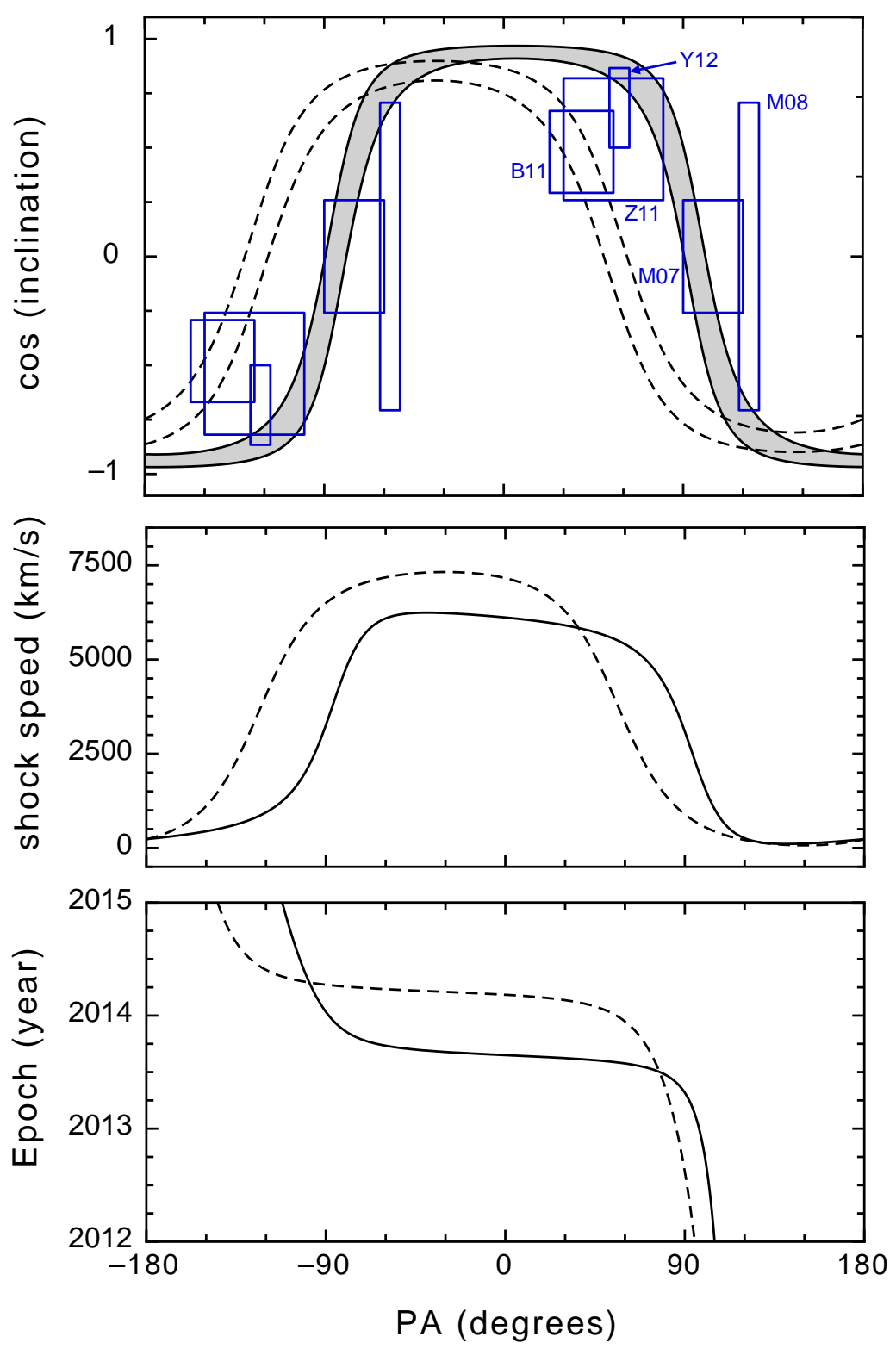

Fig. 4. - The upper panel shows the possible orientations of a Sgr A* jet directed within $5^{\circ}$ of the G2 orbits inferred by Gillessen et al. (2013) (shaded) and Phifer et al. (2013) (dashed lines). PA is measured $\mathrm{E}$ of $\mathrm{N}$ and inclination is measured relative to the line of sight towards the observer. The rectangular regions indicate possible jet orientations based on Sgr A* emission models or jet-like features identified in radio and X-rays (see text). The center and lower panels show the PA-dependence of the shock speed and interaction epoch, respectively, for the G2 orbits determined by Gillessen et al. (2013; solid lines) and Phifer et al. (2013; dashed lines). 